\title{
Association of lipid accumulation product with chronic kidney disease in Chinese community adults: a report from the REACTION study
}

Pijun Yan ${ }^{1} \mathbb{D}$, Yong $X u^{1}$, Ying Miao ${ }^{1}$, Qian Tang ${ }^{1}$, Yuru Wu ${ }^{1}$, Xue Bai ${ }^{1}$, Zhihong Zhang ${ }^{2}$, Qian $\mathrm{Li}^{3}$ and Qin Wan ${ }^{1 *}$

\begin{abstract}
Background: Limited studies regarding the correlation of lipid accumulation product (LAP) with a decreased estimated glomerular filtration rate (eGFR) have yielded conflicting findings, and no report has demonstrated the relationship of LAP with chronic kidney disease (CKD), defined as the presence of albuminuria and/or a decreased eGFR. The purpose of this study was to estimate the possible correlation of LAP with CKD prevalence in Chinese community adults.
\end{abstract}

Method: In this cross-sectional study, LAP level of 7202 participants (age $\geq 40$ years) was determined, and its possible association with CKD was evaluated by a multiple logistic regression model.

Results: Compared with subjects with non-CKD, non-albuminuria, and high eGFR, LAP levels significantly increased in female not male subjects with CKD, albuminuria, and low eGFR, respectively (all $P<0.001$ ). The univariate logistic regression analysis revealed that $L A P$ level of female not male subjects were significantly and positively associated with the prevalence of CKD $(P<0.001)$. The multivariate logistic regression analysis showed that the risk of CKD prevalence in female not male subjects progressively increased across LAP quartiles ( $P$ for trend $<0.01$ ), and the risk of CKD prevalence of subjects in Q4 significantly increased compared to those in Q1 after adjustment for potential confounding factors in Models 4 (odds ratio [OR]: 1.382, 95\% confidence intervals [Cl] 1.002-1.906, $P<0.05$ ). Stratified analysis revealed positive associations of LAP quartiles with risk of CKD prevalence in people with the following characteristics: women, older, overweight, with hypertension, normal glucose tolerance, appropriate lowdensity lipoprotein cholesterol, nonsmokers, nondrinkers, and no cardiovascular disease events.

Conclusions: High LAP levels might be significantly associated with risk of CKD prevalence in community-dwelling Chinese female adults, which may inform both public health recommendations and clinical practice.

Keywords: Lipid accumulation product, Chronic kidney disease, Visceral obesity, Albuminuria, Estimated glomerular filtration rate, Chinese community adults, Stratified analysis

\footnotetext{
* Correspondence: wanqin231@126.com

'Department of Endocrinology, the Affiliated Hospital of Southwest Medical University, Luzhou 646000, Sichuan, China

Full list of author information is available at the end of the article
}

(c) The Author(s). 2021 Open Access This article is licensed under a Creative Commons Attribution 4.0 International License, which permits use, sharing, adaptation, distribution and reproduction in any medium or format, as long as you give appropriate credit to the original author(s) and the source, provide a link to the Creative Commons licence, and indicate if changes were made. The images or other third party material in this article are included in the article's Creative Commons licence, unless indicated otherwise in a credit line to the material. If material is not included in the article's Creative Commons licence and your intended use is not permitted by statutory regulation or exceeds the permitted use, you will need to obtain permission directly from the copyright holder. To view a copy of this licence, visit http://creativecommons.org/licenses/by/4.0/ The Creative Commons Public Domain Dedication waiver (http://creativecommons.org/publicdomain/zero/1.0/) applies to the data made available in this article, unless otherwise stated in a credit line to the data. 


\section{Background}

Chronic kidney disease (CKD), a chronic condition presented as kidney structural or functional abnormalities caused by multiple factors, has been reported to be a global public health issue with an increasing incidence worldwide [1]. It is estimated that CKD impacts $~ 10 \%$ of the world's population [2] and $11.6 \%$ of the Chinese adult population in 2018 [3]. Emerging studies have shown that CKD is related to an increased risk of unfavourable health outcomes, such as cognitive impairment, end-stage renal disease, and atherosclerotic cardiovascular disease (CVD) morbidity and mortality $[2,4,5]$. However, CKD is initially often silent without warning, and a considerable proportion of patients have already developed CVD when CKD is diagnosed. Therefore, the availability of a simple, noninvasive and reliable index for the early identification of individuals who were at high risk for CKD and further determination of effective strategies to prevent its development and progression are of great significance.

Compelling data indicate that obesity, especially central obesity, is involved in the development and progression of CKD and its components, including a high urinary albumin-creatinine ratio (ACR) of $\geq 30 \mathrm{mg} / \mathrm{g}$, also called albuminuria, and a low estimated glomerular filtration rate (eGFR) of $<60 \mathrm{~mL} / \mathrm{min} / 1.73 \mathrm{~m} 2$ [5-8], but the mechanisms underlying the associations are multifactorial and still unclear. It has been well established that visceral adipose tissue (VAT) can secrete several proinflammatory cytokines and adipocytokines that lead to inflammation, oxidative stress, insulin resistance, and endothelial dysfunction and subsequently induce kidney structure and function changes such as glomerular sclerosis and albuminuria, ultimately resulting in renal dysfunction [6, 9-11]; thus, the cardiometabolic risks of VAT are higher than subcutaneous adipose tissue (SAT) [6]. In routine clinical practice, the magnetic resonance imaging (MRI) and/or computed tomography (CT), which are considered the gold standard to directly measure VAT, are not feasible because these techniques are time-consuming, costly, and radioactive [9]. Waist circumference (WC), a practical anthropometric parameter to identify central obesity, is unable to distinguish between VAT and SAT $[6,9]$. In recent years, the lipid accumulation product (LAP), calculated as a combination of fasting triglycerides (TG) and $\mathrm{WC}$, has been considered as a novel surrogate marker of central adiposity and alternative continuous index of central lipid accumulation $[11,12]$. Given the close associations between central adiposity and CKD, it is plausible that LAP might be significantly associated with CKD. However, limited studies regarding the relationship between LAP and low eGFR in specific targeted populations have yielded conflicting findings [11, 13-15]. Several studies showed that
LAP was significantly associated with low eGFR in Korean adults, rural populations in Northeast China, and populations in East China [11, 13, 14]; however, other study indicated that LAP quartiles had no significant association with the incidence of low eGFR among Iranian urban residents without diabetes [15]. Moreover, the relationship of LAP with CKD defined as low eGFR and/or albuminuria [3] has not yet been investigated.

Thus, this cross-sectional study, which was developed from the longitudinal Risk Evaluation of Cancers in Chinese Diabetic Individuals (REACTION) study, aimed to explore the correlation of LAP with CKD prevalence in the general population in China.

\section{Methods}

\section{Study population}

This current study was developed from the REACTION study, which is a national, multicentre study led by RuiJin Hospital affiliated with Shanghai Jiao-Tong University School of Medicine investigating the correlation of prediabetes and type 2 diabetes mellitus (T2DM) with cancer risk in the Chinese population [16-18]. The present study population was from Luzhou, Sichuan Province. Between May and December 2011, 10,150 participants (age $\geq 40$ years) were initially recruited. Participants with type 1 diabetes mellitus, liver disease, cancer, acute inflammatory or infectious disease, those using angiotensin receptor blocker (ARB)/angiotensin-converting enzyme inhibitors (ACEIs) drugs, anti-inflammatory and anti-infective drugs, systemic glucocorticoids, lipidlowering drugs, and those with incomplete demographic or clinical characteristics data were excluded (Fig. 1). Overall, the final analysis included 7202 subjects.

The present study was conducted in accordance with the guidelines set out in the Declaration of Helsinki, and the Research Ethics Committees of Rui-Jin Hospital affiliated with Jiao-Tong University School of Medicine approved all procedures associated with human participants (clearance no. for ethical approval. 201,114). All participants made an informed written consent to participate.

\section{Data collection and measurements}

An in-person interview and standard questionnaire was used by Trained study personnel in local community clinics to obtain data on participants' demographic information (age and sex), lifestyle behaviours (smoking status and alcohol intake), family and personal medical history, and medication use. According to cigarette smoking habits, smoking status was classified as never, former and current smokers [18]. The study participants were also categorized as never, former and current drinkers based on the type and frequency of alcohol consumption recorded [17, 18]. Before breakfast, body 


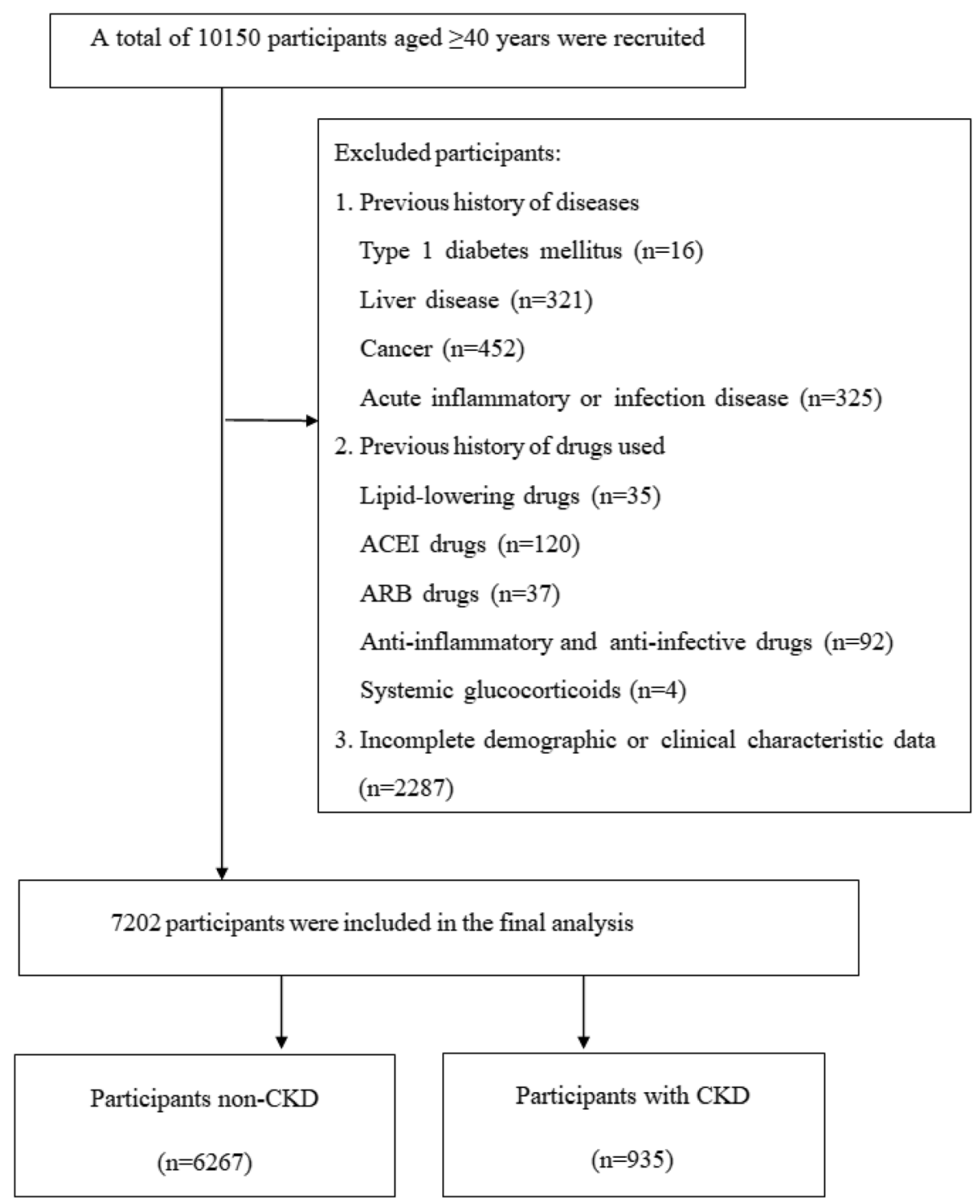

Fig. 1 Flow chart of the selection of study participants

weight, height and $\mathrm{WC}$ of the participants were measured, and then the body mass index (BMI) was obtained based on height and body weight $[16,17]$. The LAP index was calculated for women [TG $\times(\mathrm{WC}-58)]$ and men [TG $\times(\mathrm{WC}-65)]$, respectively $[11,12]$. According to the LAP index, the whole study participants were grouped into quartiles: Q1, LAP < 16.20; Q2, $16.20 \leq$ LAP < 28.88; Q3, $28.88 \leq$ LAP $\leq 50.16$; Q4, LAP > 50.16. Moreover, the subjects were also grouped into quartiles based on the LAP index: Q1, LAP < 13.69; Q2, $13.69 \leq$ LAP < 25.79; Q3, $25.79 \leq$ LAP $\leq 46.77$; Q4, LAP > 46.77 for male subjects, and Q1, LAP < 17.43; Q2, $17.43 \leq$ LAP $<30.35$; Q3, $30.35 \leq \mathrm{LAP} \leq 51.48$; Q4, LAP > 51.48 for female subjects. Systolic blood pressure (SBP) and diastolic blood pressure (DBP) were measured in all participants in a seated position three times at 5-min intervals, and then the average of the three readings was used.

A $75 \mathrm{~g}$ oral glucose tolerance test was performed in the participants after overnight fasting for at least 10 $\mathrm{h}$, and blood samples were obtained by centrifugation at 0 and $2 \mathrm{~h}$ and stored at $-80^{\circ} \mathrm{C}$. Blood glucose [glycated haemoglobin A1C (HbA1c), fasting blood glucose (FBG), 2-h postload blood glucose (PBG)], lipid profiles, including low-density and high lipoprotein cholesterol (LDL-C, HDL-C), total cholesterol (TC), $\mathrm{TG}$, and creatinine $(\mathrm{Cr})$ were measured according to relevant protocols and guidelines at a certified central laboratory, which is accredited in accordance with the International Organization for Standardization (ISO) 15,189 . 
Urinary albumin concentration and $\mathrm{Cr}$ levels of first morning urine specimens were determined using chemiluminescence immunoassay, and then the urinary ACR $(\mathrm{mg} / \mathrm{g} \mathrm{Cr}$ ) was calculated. According to the equation of Japanese coefficient-modified Chronic Kidney Disease Epidemiology Collaboration (CKD-EPI), the eGFR was calculated $[2,11]$.

\section{Definition of variables}

According to the Working Group for Obesity in China (WGOC) criteria, BMI $\geq 28 \mathrm{~kg} / \mathrm{m} 2$ was categorized as obesity, and $24 \leq \mathrm{BMI}<28 \mathrm{~kg} / \mathrm{m} 2$ was classified as overweight [19]. According to previously published criteria, the subjects were grouped into three groups: high (LDL$\mathrm{C} \geq 4.1 \mathrm{mmol} / \mathrm{L})$, borderline high $(3.4 \mathrm{mmol} / \mathrm{L} \leq \mathrm{LDL}-$ $\mathrm{C}<4.1 \mathrm{mmol} / \mathrm{L}$ ), and appropriate (LDL-C $<3.4 \mathrm{mmol} / \mathrm{L}$ ) [20]. Diagnosis of normal glucose tolerance (NGT), prediabetes, and T2DM was based on previously published criteria [16, 17]. Hypertension and CVD events [coronary heart disease (CHD), stroke, myocardial infarction (MI), and peripheral arterial disease (PAD)] were diagnosed as described previously [11, 16-18, 20, 21].

\section{Statistical analysis}

All data in the present study was analyzed by Statistical Package for Social Sciences (SPSS) version 20.0. According to the distribution of variables, continuous variables are expressed as the mean \pm standard deviation (SD) and median (25th, 75th percentile), respectively. Categorical variables are presented as numbers (percentages).

Comparisons between two groups were conducted with X2 test for categorical variables, Student's t test or MannWhitney $U$ test for continuous variables. The correlation of LAP levels and other variables with the risk of CKD prevalence were determined by univariate logistic regression analyses in men and women, respectively. Then, multivariate-adjusted logistic regression analysis was used to investigate the correlation of LAP quartiles with the risk of CKD prevalence in men and women, respectively. Odds ratio (OR) and corresponding 95\% confidence interval (CI) were calculated. Stratified analyses were conducted among different gender, age, BMI, current smoking and drinking status, prevalence of CVD events,glucose metabolism state, blood pressure, and LDL-C subgroups to investigate the relevant factors underlying the correlation of LAP with CKD prevalence. Potentialinteractions of LAP quartiles and strata variables were assessed.

Two-tailed $P$ values $<0.05$ were considered to have statistical significance.

\section{Results}

Demographic and clinical characteristics of the study population

Table 1 revealed the study population basic characteristics in men and women, respectively. In male subjects, people with CKD had significantly more users of hypoglycaemic drugs, fewer current drinkers, older age, poorer kidney function (elevated serum $\mathrm{Cr}$ and urinary ACR, and reduced eGFR), higher SBP, TC, LDL-C, blood glucose (FBG, PBG, and HbA1c), prevalence of hypertension, T2DM, CVD events (CHD and PAD), and lower height compared with those without $(P<0.001$ or $P<$ 0.01 or $P<0.05$; Table 1 and Fig.2). In female subjects, people with CKD had also significantly more users of hypoglycaemic drugs, older age, poorer kidney function (elevated serum $\mathrm{Cr}$ and urinary $\mathrm{ACR}$, and reduced eGFR), higher LAP, WC, weight, BMI, TG, blood pressure (SBP, DBP) and glucose (FBG, PBG, and HbA1c), prevalence of obesity, hypertension, T2DM, CVD events (CHD and PAD), and lower HDL-C compared with those without $(P<0.001$ or $P<0.01$ or $P<0.05$; Table 1 and Fig. 2). Further analysis indicated that only female but not male subjects with albuminuria and low eGFR displayed higher levels of LAP than the corresponding controls (all $P<0.001$, Fig. 2).

\section{Univariate analysis of variables contributing to CKD prevalence}

The prevalence of CKD showed a significantly positive correlation with age, blood pressure, TC, LDL-C, serum $\mathrm{Cr}$, urinary ACR, prevalence of T2DM and CVD events, and use of hypoglycaemic drugs, but the correlation between CKD prevalence and eGFR was negative in male subjects $(P<0.001$ or $P<0.01$ or $P<0.05$; Table 2$)$. In female subjects, the prevalence of $C K D$ was significantly and positively associated with age, BMI, WC, LAP, blood pressure, TG, serum $\mathrm{Cr}$, urinary ACR,prevalence of T2DM and CVD events, use of hypoglycaemic drugs, and negatively with HDL-C and eGFR $(P<0.001$ or $P<$ 0.01 or $P<0.05$; Table 2 ).

\section{Association of LAP quartiles with the risk of CKD prevalence}

Table 3 revealed the correlation of LAP quartiles with the risk of CKD prevalence in men and women, respectively. The results revealed that this association of LAP quartiles with the risk of CKD prevalence in men was weakened and lost significance after adjustment for age, BMI, smoking, drinking, prevalence of CVD events, blood pressure, hypoglycemic drugs, prevalence of T2DM, and LDL-C in Model 4 ( $P$ for trend $>0.05$ ). In women, the risk of CKD prevalence increased by $28.2 \%$ (95\% CI 19.8-37.2\%; $P<0.001$ ) per SD increase in LAP levels, and this association was weakened but remained significant after adjustment for multiple variables in Model 2-4 (all $P<0.001$ ). Consistently, the risk of CKD prevalence progressively increased across LAP quartiles ( $P$ for trend $<0.001$ or $P$ for trend $<0.01$ ), and the risk of CKD prevalence of subjects in Q4 significantly 
Table 1 Clinical and biochemical characteristics of study population

\begin{tabular}{|c|c|c|c|c|c|c|}
\hline & Men & & & Women & & \\
\hline & non-CKD $(n=2011)$ & CKD $(n=302)$ & $P$ & non-CKD $(n=4256)$ & CKD $(n=633)$ & $P$ \\
\hline Age (years) & $59.00(53.00-66.00)$ & $64.00(56.00-72.00)$ & $<0.001$ & $57.00(54.60-64.90)$ & $61.00(54.00-70.00)$ & $<0.001$ \\
\hline WC $(\mathrm{cm})$ & $85.30 \pm 9.85$ & $86.24 \pm 9.51$ & 0.120 & $81.00(75.00-87.20)$ & $83.00(77.00-90.00)$ & $<0.001$ \\
\hline LAP & $25.41(13.58-45.72)$ & $28.12(16.30-54.49)$ & 0.117 & $29.16(17.00-49.30)$ & $38.74(21.75-67.42)$ & $<0.001$ \\
\hline Height (cm) & $164.10(160.00-168.90)$ & 163.20 (159.00-168.00) & 0.027 & $154.10(150.20-158.00)$ & $154.00(150.00-158.00)$ & 0.081 \\
\hline Weight (kg) & $64.00(57.50-71.20)$ & $64.00(58.00-71.00)$ & 0.970 & $56.00(50.90-62.00)$ & $56.50(51.50-63.00)$ & 0.014 \\
\hline $\mathrm{BMI}\left(\mathrm{kg} / \mathrm{m}^{2}\right)$ & $23.80(21.63-26.03)$ & $24.02(22.03-25.99)$ & 0.173 & $23.53(21.43-25.86)$ & 24.19 (21.78-26.55) & $<0.001$ \\
\hline SBP $(\mathrm{mmHg})$ & $124.33(112.00-138.67)$ & 135.00 (120.00-149.00) & $<0.001$ & $120.00(107.67-134.00)$ & $128.67(114.33-145.50)$ & $<0.001$ \\
\hline $\mathrm{DBP}(\mathrm{mmHg})$ & 77.67 (70.67-85.00) & 79.00 (71.00-90.00) & 0.102 & $74.00(67.67-80.67)$ & $76.67(69.33-85.00)$ & $<0.001$ \\
\hline TC $(\mathrm{mmol} / \mathrm{L})$ & $4.51 \pm 1.08$ & $4.69 \pm 1.09$ & 0.010 & $4.82 \pm 1.10$ & $4.90 \pm 1.21$ & 0.117 \\
\hline TG $(\mathrm{mmol} / \mathrm{L})$ & $1.28(0.90-1.87)$ & $1.35(0.95-2.08)$ & 0.132 & $1.29(0.91-1.88)$ & $1.52(1.07-2.19)$ & $<0.001$ \\
\hline $\mathrm{HDL}-\mathrm{C}(\mathrm{mmol} / \mathrm{L})$ & $1.17(0.97-1.38)$ & $1.19(0.99-1.36)$ & 0.576 & $1.32(1.10-1.56)$ & $1.26(1.04-1.49)$ & $<0.001$ \\
\hline LDL-C (mmol/L) & $2.52(2.00-3.09)$ & $2.70(2.14-3.23)$ & 0.007 & $2.66(2.12-3.23)$ & $2.66(2.12-3.32)$ & 0.825 \\
\hline FBG $(\mathrm{mmol} / \mathrm{L})$ & $5.49(5.13-6.12)$ & $5.63(5.21-6.70)$ & 0.001 & $5.39(5.07-5.89)$ & $5.53(5.18-6.31)$ & $<0.001$ \\
\hline PBG (mmol/L) & $7.92(6.37-10.70)$ & $8.70(6.88-12.72)$ & $<0.001$ & $7.51(6.26-9.83)$ & $8.58(6.87-11.99)$ & $<0.001$ \\
\hline $\mathrm{HbA1c}(\%)$ & $5.90(5.60-6.30)$ & $6.00(5.70-6.60)$ & 0.001 & $5.90(5.60-6.30)$ & $6.00(5.60-6.50)$ & $<0.001$ \\
\hline $\mathrm{Cr}(\mu \mathrm{mol} / \mathrm{L})$ & $71.90(65.40-81.30)$ & 78.75 (68.88-106.20) & $<0.001$ & $59.80(54.60-64.90)$ & $63.20(55.35-81.65)$ & $<0.001$ \\
\hline $\mathrm{eGFR}\left(\mathrm{mL} / \mathrm{min} / 1.73 \mathrm{~m}^{2}\right)$ & $96.65(88.30-103.89)$ & $88.83(59.23-100.20)$ & $<0.001$ & $97.17(89.16-104.19)$ & $90.72(63.51-100.66)$ & $<0.001$ \\
\hline Urinary ACR (mg/g) & $7.74(5.08-12.30)$ & 39.30 (31.54-56.69) & $<0.001$ & $6.61(4.32-11.16)$ & 43.75 (31.79-61.89) & $<0.001$ \\
\hline Current smoker (\%) & 794 (39.48\%) & $106(35.10 \%)$ & 0.145 & $65(1.53 \%)$ & $11(1.74 \%)$ & 0.690 \\
\hline Current drinkers (\%) & 1085 (53.95\%) & $144(47.68 \%)$ & 0.042 & $643(15.11 \%)$ & 79 (12.48\%) & 0.082 \\
\hline Overweight (\%) & $730(36.30 \%)$ & 119 (39.40\%) & 0.297 & 1460 (34.30\%) & $232(36.65 \%)$ & 0.247 \\
\hline Obesity (\%) & 223 (11.09\%) & $33(10.93 \%)$ & 0.933 & 448 (10.53\%) & $100(15.80 \%)$ & $<0.001$ \\
\hline Prediabetes (\%) & 581 (28.89\%) & 76 (25.17\%) & 0.181 & 1163 (27.33\%) & $190(30.02 \%)$ & 0.158 \\
\hline T2DM (\%) & 537 (26.70\%) & $113(37.42 \%)$ & $<0.001$ & 854 (20.07\%) & $210(33.18 \%)$ & $<0.001$ \\
\hline Users of hypoglycemic drugs (\%) & 195 (9.70\%) & $46(15.23 \%)$ & 0.003 & $299(7.03 \%)$ & $84(13.27 \%)$ & $<0.001$ \\
\hline Hypertension (\%) & $726(36.10 \%)$ & $163(53.97 \%)$ & $<0.001$ & $1117(26.25 \%)$ & $316(49.92 \%)$ & $<0.001$ \\
\hline MI (\%) & $13(0.65 \%)$ & $5(1.66 \%)$ & 0.063 & $8(0.19 \%)$ & $3(0.47 \%)$ & 0.157 \\
\hline CHD (\%) & $68(3.38 \%)$ & $18(5.96 \%)$ & 0.030 & $107(2.51 \%)$ & $39(6.16 \%)$ & $<0.001$ \\
\hline Stroke (\%) & $15(0.75 \%)$ & $4(1.32 \%)$ & 0.299 & $16(0.38 \%)$ & $3(0.47 \%)$ & 0.712 \\
\hline PAD (\%) & $1(0.05 \%)$ & $3(0.99 \%)$ & $<0.001$ & $2(0.05 \%)$ & $2(0.32 \%)$ & 0.027 \\
\hline CVD (\%) & $88(4.38 \%)$ & $26(8.61 \%)$ & 0.002 & 129 (3.03\%) & $45(7.11 \%)$ & $<0.001$ \\
\hline
\end{tabular}

Data are mean \pm SD. SD Standard deviation, WC Waist circumference, LAP Lipid accumulation product, BMI Body mass index, SBP Systolic blood pressure, DBP Diastolic blood pressure, TC Total cholesterol, TG Triglyceride, HDL-C High-density lipoprotein cholesterol, LDL-C Low-density lipoprotein cholesterol, FBG Fasting blood glucose, $P B G 2 \mathrm{~h}$ postload blood glucose, HbA1c Glycated hemoglobin A1c, $C r$ Creatinine, eGFR Estimated glomerular filtration rate, ACR Albumin-tocreatinine ratio, T2DM, type 2 diabetes mellitus, MI Myocardial infarction, CHD Coronary heart disease, PAD Peripheral arterial disease, CVD Cardiovascular disease

increased compared to those in Q1 in Models $1-4(P<$ 0.001 or $P<0.01$ or $P<0.05)$.

\section{Association of LAP quartiles with risk CKD prevalence in a stratified analysis}

The results showed that the associations of higher LAP quartiles with an increased risk of CKD prevalence were not consistently the same (Table 4). A significant correlation between higher LAP quartiles and an elevated risk of CKD prevalence was only detected in subjects who were women, aged $\geq 60$ years, overweight, having NGT, hypertension, appropriate LDL-C, and no smoking, drinking, and CVD events $(P<0.01$ or $P<0.05)$. Furthermore, an interaction of blood pressure stratification with LAP quartiles was found in CKD ( $P$ for interaction < $0.001)$.

\section{Discussion}

This is currently the first study to show an increased levels of LAP in community-dwelling Chinese female 
A

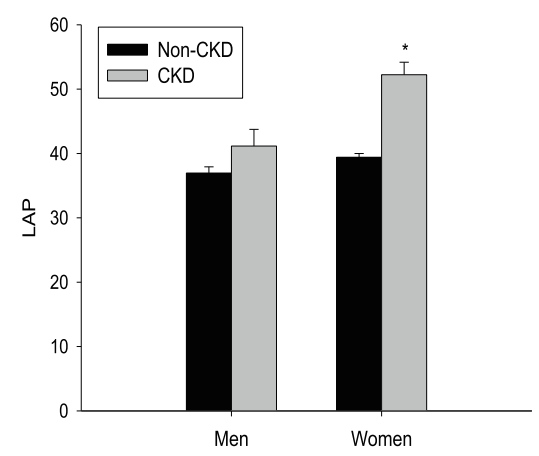

$\mathrm{C}$

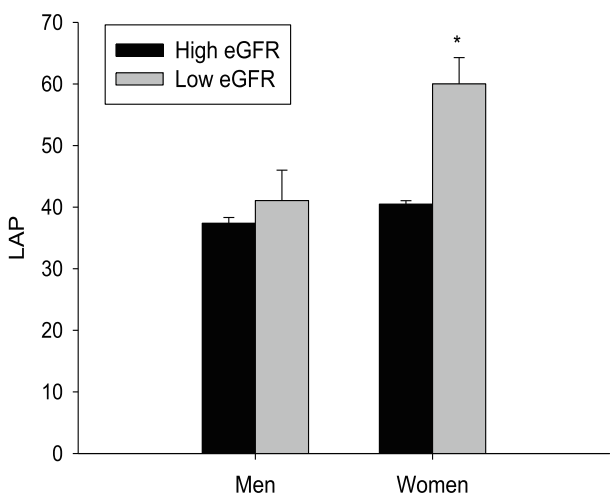

B

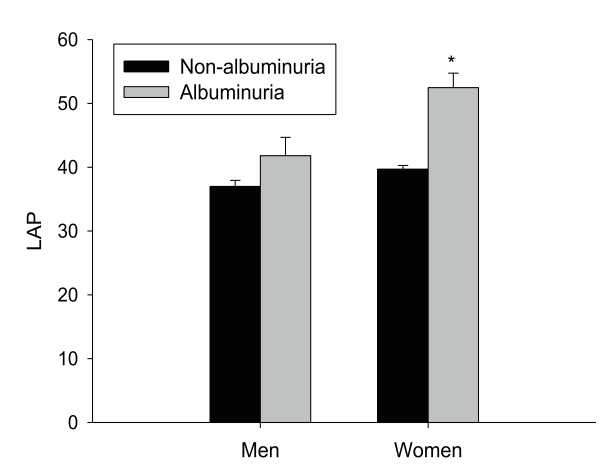

Fig. 2 LAP levels in male and female subjects by CKD, albuminuria, and low eGFR, respectively. A. LAP levels in male and female subjects by CKD, respectively. B. LAP levels in male and female subjects by albuminuria, respectively. C. LAP levels in male and female subjects by low eGFR, respectively. Vs. non-CKD group or non-albuminuria group or high eGFR, ${ }^{*} P<0.001$

not male adults with CKD, defined as low eGFR and/or the presence of albuminuria. The multivariate logistic regression analysis revealed that the risk of CKD prevalence in female not male subjects progressively increased across LAP quartiles, and the risk of CKD prevalence of subjects in Q4 significantly increased compared to those in Q1 after adjustment for potential confounding factors. Additionally, stratified analysis showed that subjects with higher LAP quartiles had an increased risk of CKD prevalence than those with lower quartiles, especially in women, people who were older, overweight, with hypertension, NGT, appropriate LDL-C, and without smoking, drinking, and CVD events.

There is evidence to suggest that obesity, especially central obesity, is an important risk factor for CKD [69]. LAP, an available and emerging surrogate marker of central obesity, can be used to assess the visceral adiposity distribution and reflect visceral adiposity dysfunction
$[11,12]$, and thus, it might be involved in the development and progression of CKD. Previous cross-sectional studies regarding the relationship of LAP with CKD (simply defined by low eGFR but not evaluated by albuminuria) have yielded conflicting findings [11, 13, 14]. An observational study in East China demonstrated that in 10,012 male and female subjects aged $\geq 18$ years, LAP levels displayed a strong association with low eGFR and could predict the risk of renal dysfunction [13]. Another study of 11,192 individuals aged $\geq 35$ years also reported a significant association of LAP with low eGFR, and LAP could predict low eGFR only in women in rural population of northeast China [14]. Seong and colleagues has recently demonstrated that LAP quartiles were association with the prevalence of low eGFR in 4947 Korean subjects (aged $\geq 20$ years); however, the association was observed only in men after adjusting for related variables [11]. The present study was consistent with those 
Table 2 Univariate analysis of variables contributing to CKD in study population

\begin{tabular}{|c|c|c|c|c|c|c|}
\hline & Men & & & Women & & \\
\hline & Univari & lysis & & & & \\
\hline & B & OR $(95 \% \mathrm{Cl})$ & $P$-value & B & OR $(95 \% \mathrm{Cl})$ & $P$-value \\
\hline Age & 0.039 & $1.040(1.027-1.053)$ & $<0.001$ & 0.046 & $1.047(1.038-1.056)$ & $<0.001$ \\
\hline BMI & 0.020 & $1.020(0.989-1.053)$ & 0.206 & 0.031 & $1.032(1.014-1.051)$ & 0.001 \\
\hline WC & 0.010 & $1.010(0.998-1.022)$ & 0.120 & 0.024 & $1.024(1.016-1.033)$ & $<0.001$ \\
\hline LAP & 0.002 & 1.002 (0.999-1.004) & 0.119 & 0.006 & $1.006(1.005-1.008)$ & $<0.001$ \\
\hline SBP & 0.020 & $1.020(1.014-1.027)$ & $<0.001$ & 0.022 & $1.022(1.018-1.026)$ & $<0.001$ \\
\hline DBP & 0.013 & $1.013(1.003-1.024)$ & 0.015 & 0.027 & 1.027 (1.019-1.035) & $<0.001$ \\
\hline TC & 0.144 & $1.155(1.035-1.290)$ & 0.010 & 0.064 & 1.066 (0.990-1.148) & 0.092 \\
\hline TG & 0.041 & $1.041(0.964-1.125)$ & 0.302 & 0.183 & $1.201(1.135-1.270)$ & $<0.001$ \\
\hline $\mathrm{HDL}-\mathrm{C}$ & 0.093 & $1.098(0.756-1.595)$ & 0.624 & -0.481 & $0.618(0.482-0.792)$ & $<0.001$ \\
\hline LDL-C & 0.194 & $1.215(1.050-1.405)$ & 0.009 & 0.013 & $1.013(0.916-1.120)$ & 0.800 \\
\hline $\mathrm{Cr}$ & 0.046 & 1.047 (1.039-1.055) & $<0.001$ & 0.056 & $1.058(1.050-1.065)$ & $<0.001$ \\
\hline eGFR & -0.050 & $0.951(0.943-0.958)$ & $<0.001$ & -0.050 & $0.951(0.946-0.956)$ & $<0.001$ \\
\hline Urinary ACR & 0.193 & $1.213(1.189-1.237)$ & $<0.001$ & 0.186 & $1.204(1.188-1.220)$ & $<0.001$ \\
\hline Drinking & -0.201 & $0.818(0.638-1.048)$ & 0.111 & -0.214 & $0.808(0.629-1.037)$ & 0.094 \\
\hline Smoking & -0.110 & $0.896(0.695-1.155)$ & 0.396 & 0.323 & $1.381(0.758-2.514)$ & 0.291 \\
\hline T2DM & 0.495 & $1.641(1.274-2.114)$ & $<0.001$ & 0.682 & 1.978 (1.649-2.372) & $<0.001$ \\
\hline Hypoglycemic drugs & 0.515 & $1.673(1.183-2.367)$ & 0.004 & 0.706 & $2.205(1.564-2.621)$ & $<0.001$ \\
\hline CVD events & 0.722 & $2.059(1.306-3.246)$ & 0.002 & 0.895 & $2.448(1.725-3.475)$ & $<0.001$ \\
\hline
\end{tabular}

Beta is the standardized coefficient and measures the influence of each variable on CKD; OR is the odds ratio and refers to the risk of CKD

reports of Zhang et al. and Dai et al. [13, 14], but was inconsistent with the findings reported by Seong et al. [11], altogether suggesting that high LAP might be associated with CKD prevalence. These discrepancies between the current study and previous cross-sectional studies might be due to differences in population characteristics such as age and male/female proportion, study groups, region, races, dietary habits, CKD definition, sample sizes, and confounding factors adjusted in the analyses. Surprisingly, the results from a prospective study, consisting of 6693 Iranian non-diabetic adults aged $\geq 18$ years, revealed that eGFR progressively decreased across LAP tertiles at baseline, but LAP was not a significant predictor of incident renal function decline in either sex after adjusting for multiple confounders [15], which was inconsistent with the present study. These discrepancies between the two studies might be due to differences in population characteristics such as age and male/female proportion, study groups, study designs, CKD definition, races, dietary habits, statistical methods, and confounding factors adjusted in the analyses. Additional studies are needed to confirm the present findings.

Interestingly, the present study revealed a significant sex difference in the effect of LAP on CKD prevalence because female but not male subjects with CKD, albuminuria, and low eGFR had significantly higher LAP levels compared with the respective control groups. Moreover, the logistic regression analysis revealed that LAP level of female not male subjects were significantly and positively associated with CKD prevalence, and the risk of CKD prevalence in female not male subjects progressively increased across LAP quartiles after adjustment for potential confounding factors. Further stratification by sex revealed that a significant correlation between higher LAP quartiles and risk of CKD prevalence was only observed in women, whereas such a relationship was not detected in men after multivariable adjustment, consistent with a previous study [14]. These findings suggest a sex-specific association of LAP with CKD prevalence, the underlying mechanisms of which might be attributed to differences in sex hormones, fat distribution, and lifestyles between men and women. The vast majority of women in the current study were > 50 years of age and thus presumably in the perimenopausal or postmenopausal stage with significantly decreased production of oestrogen, which would lead to adipose tissue redistribution characterized by more abdominal VAT, secreting various bioactive adipocytokines and inflammatory proteins, and contributing to changes in renal haemodynamics, renal vascular damage, glomerular sclerosis, renal fibrosis, low eGFR, albuminuria, and CKD [7, 22, 23]. Conversely, male subjects tended to be physically active and to consume more green tea, 
Table 3 Association between LAP quartiles and risk of CKD in the study population

\begin{tabular}{|c|c|c|c|c|c|c|}
\hline \multirow[t]{2}{*}{ Variable } & \multirow[t]{2}{*}{ Per SD increase } & \multicolumn{4}{|c|}{ LAP quartiles } & \multirow{2}{*}{$\begin{array}{l}P \text { for } \\
\text { trend }\end{array}$} \\
\hline & & $\overline{Q 1}$ & Q2 & Q3 & Q4 & \\
\hline \multicolumn{7}{|l|}{ Men } \\
\hline \multicolumn{7}{|l|}{ Model 1} \\
\hline OR $(95 \% \mathrm{Cl})$ & $1.088(0.978-1.210)$ & 1 & $1.228(0.860-1.753)$ & $1.193(0.846-1.682)$ & $1.570(1.131-2.178)$ & 0.029 \\
\hline$P$-value & 0.119 & & 0.258 & 0.315 & 0.007 & \\
\hline \multicolumn{7}{|l|}{ Model 2} \\
\hline OR $(95 \% \mathrm{Cl})$ & $1.116(0.991-1.256)$ & 1 & $1.215(0.819-1.803)$ & $1.173(0.793-1.735)$ & $1.725(1.115-2.668)$ & 0.040 \\
\hline$P$-value & 0.069 & & 0.332 & 0.423 & 0.014 & \\
\hline \multicolumn{7}{|l|}{ Model 3} \\
\hline OR $(95 \% \mathrm{Cl})$ & $1.118(0.993-1.260)$ & 1 & $1.210(0.814-1.799)$ & $1.173(0.790-1.741)$ & $1.725(1.110-2.678)$ & 0.037 \\
\hline$P$-value & 0.066 & & 0.346 & 0.430 & 0.015 & \\
\hline \multicolumn{7}{|l|}{ Model 4} \\
\hline OR $(95 \% \mathrm{Cl})$ & $1.081(0.951-1.228)$ & 1 & $1.069(0.705-1.621)$ & $1.003(0.651-1.544)$ & $1.393(0.870-2.228)$ & 0.320 \\
\hline$P$-value & 0.232 & & 0.754 & 0.991 & 0.167 & \\
\hline \multicolumn{7}{|l|}{ Women } \\
\hline \multicolumn{7}{|l|}{ Model 1} \\
\hline OR $(95 \% \mathrm{Cl})$ & $1.282(1.198-1.372)$ & 1 & $1.096(0.839-1.431)$ & $1.459(1.132-1.881)$ & $2.173(1.696-2.784)$ & $<0.001$ \\
\hline$P$-value & $<0.001$ & & 0.501 & 0.003 & $<0.001$ & \\
\hline \multicolumn{7}{|l|}{ Model 2} \\
\hline OR $(95 \% \mathrm{Cl})$ & $1.224(1.137-1.318)$ & 1 & $0.979(0.738-1.299)$ & $1.157(0.869-1.540)$ & 1.599 (1.190-2.148) & $<0.001$ \\
\hline$P$-value & $<0.001$ & & 0.883 & 0.318 & 0.002 & \\
\hline \multicolumn{7}{|l|}{ Model 3} \\
\hline OR $(95 \% \mathrm{Cl})$ & $1.220(1.133-1.313)$ & 1 & $0.988(0.744-1.311)$ & $1.132(0.848-1.510)$ & $1.582(1.176-2.127)$ & $<0.001$ \\
\hline$P$-value & $<0.001$ & & 0.931 & 0.400 & 0.002 & \\
\hline \multicolumn{7}{|l|}{ Model 4} \\
\hline OR $(95 \% \mathrm{Cl})$ & $1.169(1.082-1.262)$ & 1 & $0.981(0.730-1.317)$ & $1.051(0.773-1.430)$ & $1.382(1.002-1.906)$ & 0.002 \\
\hline$P$-value & $<0.001$ & & 0.898 & 0.751 & 0.048 & \\
\hline
\end{tabular}

Data are expressed as OR $(95 \% \mathrm{Cl})+P$ value, unless stated otherwise. Model 1: unadjusted; Model 2: adjusted for age and BMl; Model 3: additionally adjusted for smoking, drinking, prevalence of CVD events based on Model 2; Model 4: additionally adjusted for SBP, DBP, hypoglycemic drugs, LDL-C, and prevalence of T2DM based on Model 3

both of which may lead to a lower distribution of abdominal fat in males compared with females at the same age who were less active and consumed less tea [24-26]. Several reports have suggested that age is an important risk factor for CKD [11]. The present study also revealed that the prevalence of CKD was significantly and positively associated with age, and also suggested a positive relationship of LAP quartiles with risk of CKD prevalence in subjects aged $\geq 60$ years but not those aged $<60$ years, which was similar to a previous study [27]. These results suggest older age might be associated with CKD prevalence because elderly individuals have higher prevalenceof comorbidities, such as obesity, hyperuricaemia, dyslipidaemia, diabetes, and hypertension, and more use of medications for these chronic diseases, which may have a profound negative impact on kidney function. Overweight/obesity has been reported to be involved in the development of CKD [5-8]. The present study also showed that female subjects with CKD had significantly higher BMI and prevalence of obesity compared with those without CKD, and BMI was positively correlated with CKD prevalence. Further stratified analysis by BMI revealed that a significant correlation between higher LAP quartiles and risk of CKD prevalence was only detected in overweight subjects, which was similar to a previous study [28], suggesting that being overweight may correlate with the prevalence of CKD via several modifiable atherosclerotic and cardiometabolic risks, as well as endothelial dysfunction. Moreover, after adjusting for sex, age, and BMI, the correlation of LAP quartiles with risk of CKD prevalence in female subjects was attenuated, indicating that female sex, older age, and overweight status added to the risk of CKD prevalence and altogether probably weakened the correlation of 
Table 4 Association between LAP quartiles and increased risk of CKD in different participants

\begin{tabular}{|c|c|c|c|c|c|c|}
\hline \multirow[t]{3}{*}{ Variable } & \multicolumn{4}{|c|}{ LAP quartiles } & \multirow{3}{*}{$\begin{array}{l}P \text { for } \\
\text { trend }\end{array}$} & \multirow{3}{*}{$\begin{array}{l}P \text { for } \\
\text { interaction }\end{array}$} \\
\hline & Q1 & Q2 & Q3 & Q4 & & \\
\hline & $\begin{array}{l}\text { OR }(95 \% C) \\
P \text {-value }\end{array}$ & $\begin{array}{l}\text { OR }(95 \% \mathrm{Cl}) \\
P \text {-value }\end{array}$ & $\begin{array}{l}\text { OR }(95 \% \mathrm{Cl}) \\
P \text {-value }\end{array}$ & $\begin{array}{l}\text { OR }(95 \% \mathrm{Cl}) \\
P \text {-value }\end{array}$ & & \\
\hline Gender & & & & & & 0.511 \\
\hline Men & 1 & $\begin{array}{l}1.269(0.851-1.892) \\
0.243\end{array}$ & $\begin{array}{l}0.912(0.584-1.425) \\
0.687\end{array}$ & $\begin{array}{l}1.472(0.905-2.394) \\
0.120\end{array}$ & 0.211 & \\
\hline Women & 1 & $\begin{array}{l}0.982(0.721-1.337) \\
0.908\end{array}$ & $\begin{array}{l}0.989(0.715-1.368) \\
0.947\end{array}$ & $\begin{array}{l}1.462(1.041-2.054) \\
0.029\end{array}$ & 0.006 & \\
\hline Age, years & & & & & & 0.061 \\
\hline$<60$ & 1 & $\begin{array}{l}1.098(0.784-1.539) \\
0.586\end{array}$ & $\begin{array}{l}1.077(0.737-1.572) \\
0.702\end{array}$ & $\begin{array}{l}1.622(1.086-2.425) \\
0.018\end{array}$ & 0.050 & \\
\hline$\geq 60$ & 1 & $\begin{array}{l}1.071(0.751-1.527) \\
0.705\end{array}$ & $\begin{array}{l}0.927(0.641-1.340) \\
0.686\end{array}$ & $\begin{array}{l}1.427(0.970-2.100) \\
0.071\end{array}$ & 0.014 & \\
\hline BMI status & & & & & & 0.064 \\
\hline Normal & 1 & $\begin{array}{l}1.096(0.828-1.451) \\
0.522\end{array}$ & $\begin{array}{l}0.914(0.648-1.289) \\
0.609\end{array}$ & $\begin{array}{l}1.288(0.861-1.926) \\
0.218\end{array}$ & 0.492 & \\
\hline Overweight & 1 & $\begin{array}{l}1.110(0.860-1.434) \\
0.422\end{array}$ & $\begin{array}{l}0.993(0.743-1.328) \\
0.963\end{array}$ & $\begin{array}{l}1.473(1.072-2.024) \\
0.017\end{array}$ & 0.002 & \\
\hline Obesity & 1 & $\begin{array}{l}1.510(0.099-23.014) \\
0.767\end{array}$ & $\begin{array}{l}4.444(0.418-47.272) \\
0.216\end{array}$ & $\begin{array}{l}2.061(0.240-17.699) \\
0.510\end{array}$ & 0.125 & \\
\hline Glucose metabolism status & & & & & & 0.636 \\
\hline NGT & 1 & $\begin{array}{l}0.868(0.624-1.208) \\
0.401\end{array}$ & $\begin{array}{l}0.923(0.633-1.345) \\
0.676\end{array}$ & $\begin{array}{l}1.697(1.117-2.577) \\
0.013\end{array}$ & 0.019 & \\
\hline Prediabetes & 1 & $\begin{array}{l}1.495(0.904-2.472) \\
0.117\end{array}$ & $\begin{array}{l}1.106(0.650-1.884) \\
0.710\end{array}$ & $\begin{array}{l}1.673(0.943-2.969) \\
0.078\end{array}$ & 0.592 & \\
\hline T2DM & 1 & $\begin{array}{l}1.179(0.662-2.101) \\
0.576\end{array}$ & $\begin{array}{l}0.855(0.492-1.485) \\
0.577\end{array}$ & $\begin{array}{l}1.379(0.798-2.381) \\
0.249\end{array}$ & 0.060 & \\
\hline Blood pressure status & & & & & & $<0.001$ \\
\hline Normal & 1 & $\begin{array}{l}1.061(0.793-1.421) \\
0.689\end{array}$ & $\begin{array}{l}0.932(0.672-1.294) \\
0.675\end{array}$ & $\begin{array}{l}1.568(1.088-2.259) \\
0.016\end{array}$ & 0.065 & \\
\hline Hypertension & 1 & $\begin{array}{l}1.122(0.717-1.756) \\
0.615\end{array}$ & $\begin{array}{l}1.056(0.667-1.671) \\
0.816\end{array}$ & $\begin{array}{l}1.427(0.908-2.242) \\
0.123\end{array}$ & 0.035 & \\
\hline LDL-C & & & & & & 0.255 \\
\hline Appropriate & 1 & $\begin{array}{l}1.177(0.906-1.529) \\
0.222\end{array}$ & $\begin{array}{l}1.052(0.790-1.402) \\
0.727\end{array}$ & $\begin{array}{l}1.505(1.116-2.028) \\
0.007\end{array}$ & 0.014 & \\
\hline Borderline high & 1 & $\begin{array}{l}0.622(0.265-1.461) \\
0.276\end{array}$ & $\begin{array}{l}0.788(0.354-1.753) \\
0.559\end{array}$ & $\begin{array}{l}1.908(0.787-4.622) \\
0.153\end{array}$ & 0.059 & \\
\hline High & 1 & $\begin{array}{l}0.326(0.078-1.356) \\
0.123\end{array}$ & $\begin{array}{l}0.207(0.050-0.859) \\
0.030\end{array}$ & $\begin{array}{l}0.891(0.256-3.095) \\
0.856\end{array}$ & 0.747 & \\
\hline CVD events & & & & & & 0.653 \\
\hline No & 1 & $\begin{array}{l}1.066(0.832-1.365) \\
0.613\end{array}$ & $\begin{array}{l}0.920(0.705-1.201) \\
0.539\end{array}$ & $\begin{array}{l}1.476(1.119-1.948) \\
0.006\end{array}$ & 0.007 & \\
\hline Yes & 1 & $\begin{array}{l}3.388(0.685-16.748) \\
0.134\end{array}$ & $\begin{array}{l}2.337(0.683-8.005) \\
0.176\end{array}$ & $\begin{array}{l}2.236(0.447-11.198) \\
0.328\end{array}$ & 0.166 & \\
\hline Current drinking status & & & & & & 0.640 \\
\hline No & 1 & $\begin{array}{l}1.010(0.757-1.350) \\
0.944\end{array}$ & $\begin{array}{l}0.910(0.671-1.234) \\
0.543\end{array}$ & $\begin{array}{l}1.450(1.051-2.000) \\
0.024\end{array}$ & 0.004 & \\
\hline Yes & 1 & $\begin{array}{l}1.238(0.786-1.950) \\
0.357\end{array}$ & $\begin{array}{l}1.070(0.637-1.798) \\
0.799\end{array}$ & $\begin{array}{l}1.471(0.858-2.523) \\
0.161\end{array}$ & 0.590 & \\
\hline Current smoking status & & & & & & 0.413 \\
\hline No & 1 & $\begin{array}{l}1.082(0.831-1.407) \\
0.559\end{array}$ & $\begin{array}{l}1.019(0.768-1.353) \\
0.895\end{array}$ & $\begin{array}{l}1.455(1.083-1.954) \\
0.013\end{array}$ & 0.004 & \\
\hline
\end{tabular}


Table 4 Association between LAP quartiles and increased risk of CKD in different participants (Continued)

\begin{tabular}{|c|c|c|c|c|c|c|}
\hline \multirow[t]{3}{*}{ Variable } & \multicolumn{4}{|c|}{ LAP quartiles } & \multirow{3}{*}{$\begin{array}{l}P \text { for } \\
\text { trend }\end{array}$} & \multirow{3}{*}{$\begin{array}{l}P \text { for } \\
\text { interaction }\end{array}$} \\
\hline & $\overline{\text { Q1 }}$ & Q2 & Q3 & Q4 & & \\
\hline & $\begin{array}{l}\text { OR }(95 \% \mathrm{C}) \\
P \text {-value }\end{array}$ & $\begin{array}{l}\text { OR }(95 \% \mathrm{Cl}) \\
P \text {-value }\end{array}$ & $\begin{array}{l}\text { OR }(95 \% \mathrm{Cl}) \\
P \text {-value }\end{array}$ & $\begin{array}{l}\text { OR }(95 \% \mathrm{Cl}) \\
P \text {-value }\end{array}$ & & \\
\hline Yes & 1 & $\begin{array}{l}1.011(0.525-1.946) \\
0.974\end{array}$ & $\begin{array}{l}0.705(0.355-1.400) \\
0.318\end{array}$ & $\begin{array}{l}1.847(0.848-4.026) \\
0.122\end{array}$ & 0.494 & \\
\hline
\end{tabular}

Data are expressed as OR $(95 \% \mathrm{Cl})+P$ value, unless stated otherwise. Adjusted for sex, age, BMI, smoking, drinking, CVD events, SBP, DBP, LDL-C, prevalence of T2DM, and hypoglycemic drugs

LAP quartiles with risk of CKD prevalence. Thus, it is of great importance to monitor LAP for early screening for CKD among people who were women, older, and overweight to further prevent and delay the development and progression of CKD by incorporating changes in lifestyle and necessary medical treatments.

Emerging epidemiologic studies have suggested that diabetes, hypertension, and dyslipidaemia are important risk factors for CKD [1, 4, 6, 27-29]. Thepresent study also showed that male and female subjects with CKD had significantly more users of hypoglycaemic drugs, higher blood glucose (FBG, PBG, and HbA1c), prevalence of T2DM and hypertension compared with those without CKD. Moreover, the prevalence of CKD showed a significantly positive correlation with blood pressure, prevalence of T2DM, and use of hypoglycaemic drugs in both sexes. These results suggested that the prevalence of CKD might be correlated with the prevalence of diabetes and hypertension. Additionally, the correlation of LAP quartiles with CKD prevalence in female subjects was attenuated but retained significance after further adjusting for blood pressure, LDL-C, prevalence of T2DM, and hypoglycaemic drugs in Model 4, suggesting that diabetes, hypertension, and dyslipidaemia added to the risk of CKD prevalence and probably weakened the correlation of LAP with of risk of CKD prevalence. Further stratified analysis showed that a significant correlation of higher LAP quartiles with an increased risk for CKD prevalence was only detected in hypertensive subjects but not in those with normal blood pressure, which was generally in line with several previous studies [28, 30,31 , suggesting that hypertension might be associated with the prevalence of CKD. More importantly, an interaction of hypertension with LAP on CKD prevalence was also observed, indicating that hypertension and elevated LAP may synergistically increase the risk of CKD prevalence, and hypertension might a stronger factor affecting the relationship between LAP and CKD prevalence. It is suggested that the most important lipid risk factor and therapeutic target for CVD is LDL-C [32]; however, there is still a considerable residual risk of CVD events even after a reduction of LDL-C to the recommended concentrations with statins and other lipidlowering agents [31]. It is generally accepted that CKD and CVD share common risk factors, such as diabetes mellitus and dyslipidaemia [33, 34]. Of note, stratified analysis also revealed that significant associations between higher LAP quartiles and CKD were still detected in people with NGT and appropriate LDL-C $(<3.4$ $\mathrm{mmol} / \mathrm{L}$ ) as recommended by the guidelines, indicative of an excess risk in people with NGT and appropriate LDL-C. Additionally, the risk of CKD might already be present in community-dwelling Chinese adults with NGT and appropriate LDL-C. The lack of significant associations of LAP with CKD prevalence among subjects with prediabetes, T2DM, and abnormal LDL-C is speculated to be due to the following factors: first, subjects with prediabetes, T2DM, abnormal LDL-C might be inclined to change their diet and lifestyle habits and receive related treatment, thus contributing to the favourable effect of CKD prevention. Additionally, they were individually and jointly related to a decreased risk of CKD. Second, both experimental and clinical data have identified T2DM and dyslipidaemia as important causes of CKD, so it is reasonable to speculate that the strong relationships of prediabetes, T2DM, and abnormal LDL-C with CKD probably weakens the correlation of LAP with risk of CKD prevalence. Third, the small sample size of some subgroups, especially borderline high LDL-C $(n=973)$ and high LDL-C $(n=355)$, and the large $95 \%$ CIs have resulted in data availability or imprecise estimations. Further studies with larger sample sizes and detailed information about dietary structures, lifestyle habits, and medications are needed to investigate the association of LAP with CKD in different subgroups.

Many studies have reported significant relationships of smoking, drinking, and CVD with CKD [4, 34, 35,36]. The present study showed that patients with CKD had significantly higher prevalence of CVD events (CHD and PAD) than those with non-CKD, suggesting that the prevalence of CKD might be correlated with CVD events. Moreover, a significantly positive correlation between prevalence of CKD and CVD events. Additionally, after further adjusting for smoking, drinking, and prevalence of CVD events, the correlation of LAP quartiles with risk of CKD prevalence in female subjects was attenuated but retained significance, suggesting that smoking, drinking, and CVD added to the risk of CKD 
prevalence and probably weakened the correlation of LAP with risk of CKD prevalence. However, the stratified analysis demonstrated a significant correlation of higher LAP quartiles with an increased risk for CKD prevalence in subjects without smoking, drinking, and CVD events but not those with, which be due to several factors. First, both experimental and clinical data have identified smoking, drinking, and CVD as important causes of CKD, so it is reasonable to speculate that the strong relationships of smoking, drinking and CVD events with CKD likely weakened the correlation of LAP with risk of CKD prevalence. Second, the small sample sizes of some subgroups, especially CVD events $(n=$ $288)$ and smokers $(n=976)$ and the large $95 \%$ CIs led to data availability or imprecise estimations. Further research in a larger population is needed to verify our speculation.

\section{Study strengths and limitations}

Several potential limitations exist in the present study. First, the cross-sectional study design precluded the establishment of causality, which requires confirmation in large-scale longitudinal studies. Second, although those subjects who used ACEI/ARB and lipid-lowering drugs were excluded and hypoglycaemic drugs were adjusted, it is still possible that other drugs could partially affect the correlation between LAP and CKD prevalence. Third, the population in the present study included only community-dwelling middle-aged and elderly subjects in Luzhou city located within Sichuan Province in Southwest China; therefore, the present findings may not be generalizable to other populations. Despite these limitations, the current study is not without strengths, including a standardized method of data collection, comprehensive adjustment for major traditional risk factors, and a relatively large sample size, which can strengthen the reliability of our findings. More importantly, the present study is the first to provide clinical evidence for a potential link between LAP and CKD prevalence, defined as low eGFR and/or presence of albuminuria, in Chinese community adults.

\section{Conclusions}

Higher LAP might be significantly related to risk of CKD prevalence in Chinese community female adults. Subjects with elevated LAP had a higher risk for CKD prevalence, especially those who were older, overweight, with hypertension, NGT, appropriate LDL-C, and without smoking, drinking, and CVD events. These results indicate that LAP may be a useful and reliable indicator of CKD prevalence and highlight the importance of paying more attention to CKD in middle-aged and elderly subjects with central obesity to further reduce CKDassociated unfavourable health outcomes.

\begin{abstract}
Abbreviations
CKD: Chronic kidney disease; CVD: Cardiovascular disease; BMI: Body mass index; WC: Waist circumference; LAP: Lipid accumulation product; TG: Triglyceride; eGFR: Estimated glomerular filtration rate; ACR: Albumin-tocreatinine ratio; Q: Quartile; SBP: Systolic blood pressure; DBP: Diastolic blood pressure; FBG: Fasting blood glucose; PBG: 2 h postload blood glucose; HbA1c: Glycated hemoglobin A1c; TC: Total cholesterol; HDL-C: High-density lipoprotein cholesterol; LDL-C: Low-density lipoprotein cholesterol;

Cr: Creatinine; CKD-EPI: Chronic Kidney Disease Epidemiology Collaboration; T2DM: Type 2 diabetes mellitus; NGT: Normal glucose tolerance; MI: Myocardial infarction; CHD: Coronary heart disease; PAD: Peripheral arterial disease; SD: Standard deviation; OR: Odds ratios; Cl: Confidence intervals; CT: Computed tomography; MRI: Magnetic resonance imaging; VAT: Visceral adipose tissue; SAT: Subcutaneous adipose tissue; REACTION: Risk Evaluation of Cancers in Chinese Diabetic Individuals; ACEl: Angiotensin-converting enzyme inhibitor; ARB: Angiotensin receptor blocker; ISO: International Organization for Standardization
\end{abstract}

\section{Acknowledgements}

The authors would like to thank all of our colleagues in the clinical laboratory centre and endocrine laboratory and all the nurses in our department for their hard work and valuable assistance with this study.

\section{Authors' contributions}

All authors contributed significantly to the manuscript. Pijun Yan conducted the population study, analysed and interpreted the data, and drafted and critically revised the manuscript. Yong Xu significantly revised the draft, interpreted the data, and was involved in the data analyses. Ying Miao, Qian Tang, Yuru Wu and Xue Bai conducted the study, tested the sample, collected the information and participated in the data interpretation. Zhihong Zhang and Qian Li were involved in data management and draft revision. QW is the PI of the project, who designed the study and critically revised the manuscript. All authors read and approved the final manuscript.

\section{Funding}

This study was supported by the grants from the National Key New Drug Creation and Manufacturing Program of Ministry of Science and Technology (2012ZX09303006-001).

\section{Declarations}

\section{Ethics approval and consent to participate}

The present study was conducted in accordance with the guidelines set out in the Declaration of Helsinki, and the Research Ethics Committees of Rui-Jin Hospital affiliated with Jiao-Tong University School of Medicine approved all procedures associated with human participants (clearance no. for ethical approval. 201114). All participants made an informed written consent to participate.

\section{Consent for publication}

Not applicable.

\section{Competing interests}

The authors declare that they have no competing interests.

\section{Author details}

'Department of Endocrinology, the Affiliated Hospital of Southwest Medical University, Luzhou 646000, Sichuan, China. ${ }^{2}$ Department of General Medicine, the Affiliated Hospital of Southwest Medical University, Luzhou 646000, Sichuan, China. ${ }^{3}$ Southwest Medical University, Luzhou 646000, Sichuan, China.

Received: 18 May 2021 Accepted: 26 September 2021

Published online: 09 October 2021

\section{References}

1. López-Novoa JM, Martínez-Salgado C, Rodríguez-Peña AB, López-Hernández FJ. Common pathophysiological mechanisms of chronic kidney disease: therapeutic perspectives. Pharmacol Ther. 2010;128(1):61-81. https://doi. org/10.1016/j.pharmthera.2010.05.006. 
2. Morris AP, Le TH, Wu H, Akbarov A, van der Most PJ, Hemani G, et al. Transethnic kidney function association study reveals putative causal genes and effects on kidney-specific disease aetiologies. Nat Commun. 2019;10(1):29. https://doi.org/10.1038/s41467-018-07867-7.

3. Wang F, He K, Wang J, Zhao MH, Li Y, Zhang L, et al. Prevalence and risk factors for CKD: a comparison between the adult populations in China and the United States. Kidney Int Rep. 2018;3(5):1135-43. https://doi.org/10.101 6/j.ekir.2018.05.011.

4. Bello AK, Levin A, Manns BJ, Feehally J, Drueke T, Faruque L, et al. Effective CKD care in European countries: challenges and opportunities for health policy. Am J Kidney Dis. 2015;65(1):15-25. https://doi.org/10.1053/j.ajkd.2014. 07.033.

5. Viggiano D, Wagner CA, Martino G, Nedergaard M, Zoccali C, Unwin R, et al. Mechanisms of cognitive dysfunction in CKD. Nat Rev Nephrol. 2020;16(8): 452-69. https://doi.org/10.1038/s41581-020-0266-9.

6. Wang J, Jin X, Chen K, Yan W, Wang A, Zhu B, et al. Visceral adiposity index is closely associated with urinary albumin-creatinine ratio in the Chinese population with prediabetes. Diabetes Metab Res Rev. 2020;27:e3424. https://doi.org/10.1002/dmrr.3424

7. Moh MC, Sum CF, Tavintharan S, Ang K, Kwan PY, Lee SBM, et al. Gain in adiposity over 3 years is associated with progressive renal decline in multiethnic south-east Asians with type 2 diabetes. J Diabetes. 2019;11(4):316-25. https://doi.org/10.1111/1753-0407.12848.

8. Wu IW, Liao PJ, Ting MK, Chen SW, Yang NI, Hsu KH. Combination of Thigh Circumference and Indices of Central Obesity Helps Predict Incident Chronic Kidney Disease: A 14-Year Prospective Cohort Study Using a ThreeDimensional Body Laser Scanner. J Ren Nutr. 2021;S1051-2276(21):00173-4.

9. Sun K, Lin D, Li F, Qi Y, Feng W, Ren M, et al. Visceral adiposity index is associated with increased urinary albumin excretion: a population-based study. Clin Nutr. 2019;38(3):1332-8. https://doi.org/10.1016/j.clnu.2018.05.025.

10. Su W, Wang J, Mu Y. Association between Hypertriglyceridemic waist phenotype and increased urinary albumin-creatinine ratio in Chinese adults: the REACTION study. Diabetes Metab Syndr Obes. 2020;13:2965-74. https:// doi.org/10.2147/DMSO.S257736

11. Seong JM, Lee JH, Gi MY, Son YH, Moon AE, Park CE, et al. Gender difference in the association of chronic kidney disease with visceral adiposity index and lipid accumulation product index in Korean adults: Korean National Health and nutrition examination survey. Int Urol Nephrol. 2021;53(7):1417-25. https://doi.org/10.1007/s11255-020-02735-0.

12. loachimescu AG, Brennan DM, Hoar BM, Hoogwerf BJ. The lipid accumulation product and all-cause mortality in patients at high cardiovascular risk: a PreCIS database study. Obesity (Silver Spring). 2010; 18(9):1836-44. https://doi.org/10.1038/oby.2009.453.

13. Zhang K, Li Q, Chen Y, Wang N, Lu Y. Visceral adiposity and renal function: an observational study from SPECT-China. Lipids Health Dis. 2017;16(1):205. https://doi.org/10.1186/s12944-017-0597-0.

14. Dai D, Chang Y, Chen Y, Chen S, Yu S, Guo X, et al. Visceral adiposity index and lipid accumulation product index: two alternate body indices to identify chronic kidney disease among the rural population in Northeast China. Int J Environ Res Public Health. 2016;13(12):1231. https://doi.org/10.33 90/ijerph13121231.

15. Mousapour P, Barzin M, Valizadeh M, Mahdavi M, Azizi F, Hosseinpanah F. Predictive performance of lipid accumulation product and visceral adiposity index for renal function decline in non-diabetic adults, an 8.6-year follow-up. Clin Exp Nephrol. 2020;24(3):225-34. https://doi.org/10.1007/s10157-019-01813-7.

16. Lu J, He J, Li M, Tang X, Hu R, Shi L, et al. Predictive value of fasting glucose, Postload glucose, and hemoglobin $\mathrm{A} 1 \mathrm{C}$ on risk of diabetes and complications in Chinese adults. Diabetes Care. 2019;42(8):1539-48. https:// doi.org/10.2337/dc18-1390

17. Wang T, Lu J, Su Q, Chen Y, Bi Y, Mu Y, et al. Ideal cardiovascular health metrics and major cardiovascular events in patients with prediabetes and diabetes. JAMA Cardiol. 2019;4(9):874-83. https://doi.org/10.1001/jamaca rdio.2019.2499.

18. Bi Y, Lu J, Wang W, Mu Y, Zhao J, Liu C, et al. Cohort profile: risk evaluation of cancers in Chinese diabetic individuals: a longitudinal (REACTION) study. J Diabetes. 2014:6(2):147-57. https://doi.org/10.1111/1753-0407.12108.

19. Zhou BF. Cooperative Meta-analysis Group of the Working Group on obesity in China. Predictive values of body mass index and waist circumference for risk factors of certain related diseases in Chinese adults-study on optimal cut-off points of body mass index and waist circumference in Chinese adults. Biomed Environ Sci. 2002;15(1):83-96.
20. Zhu B, Wang J, Chen K, Yan W, Wang A, Wang W, et al. A high triglyceride glucose index is more closely associated with hypertension than lipid or glycemic parameters in elderly individuals: a cross-sectional survey from the Reaction study. Cardiovasc Diabetol. 2020;19(1):112. https://doi.org/10.1186/ s12933-020-01077-6.

21. Hussein AA, Uno K, Wolski K, Kapadia S, Schoenhagen P, Tuzcu EM, et al. Peripheral arterial disease and progression of coronary atherosclerosis. J Am Coll Cardiol. 2011;57(10):1220-5. https://doi.org/10.1016/j.jacc.2010.10.034.

22. Christou GA, Kiortsis DN. The role of adiponectin in renal physiology and development of albuminuria. J Endocrinol. 2014;221(2):R49-61. https://doi. org/10.1530/JOE-13-0578.

23. Axelsson J, Bergsten A, Qureshi AR, Heimbürger $O$, Bárány P, Lönnqvist F, et al. Elevated resistin levels in chronic kidney disease are associated with decreased glomerular filtration rate and inflammation, but not with insulin resistance. Kidney Int. 2006;69(3):596-604. https://doi.org/10.1038/sj.ki. 5000089.

24. Dash SR, Hoare E, Varsamis P, Jennings GLR, Kingwell BA. Sex-specific lifestyle and biomedical risk factors for chronic disease among early-middle, middle and older aged Australian adults. Int J Environ Res Public Health. 2019;16(2):224. https://doi.org/10.3390/ijerph16020224.

25. Tian T, Lv J, Jin G, Yu C, Guo Y, Bian Z, Yang L, Chen Y, Shen H, Chen Z, Hu Z, Li L, China Kadoorie Biobank Collaborative Group Tea consumption and risk of stroke in Chinese adults: a prospective cohort study of 0.5 million men and women. Am J Clin Nutr 2020; 111(1):197-206, 1, doi: https://doi. org/10.1093/ajen/nqz274.

26. Deka A, Vita JA. Tea and cardiovascular disease. Pharmacol Res. 2011;64(2): 136-45. https://doi.org/10.1016/j.phrs.2011.03.009.

27. Qin S, Wang A, Gu S, Wang W, Gao Z, Tang X, et al. Association between obesity and urinary albumin-creatinine ratio in the middle-aged and elderly population of southern and northern China: a cross-sectional study. BMJ Open. 2021;11(1):e040214. https://doi.org/10.1136/bmjopen-2020-040214.

28. Liu Y, Wang Y, Wang J, Chen K, Jin L, Wang W, et al. Lipid accumulation product is associated with urinary albumin-creatinine ratio in Chinese Prediabitic population: a report from the REACTION study. Diabetes Metab Syndr Obes. 2021;14:2415-25. https://doi.org/10.2147/DMSO.S310751.

29. Vallianou NG, Mitesh S, Gkogkou A, Geladari E. Chronic kidney disease and cardiovascular disease: is there any relationship? Curr Cardiol Rev. 2019; 15(1):55-63. https://doi.org/10.2174/1573403X14666180711124825.

30. Xue J, Li B, Wang J, Yu S, Wang A, An P, et al. Association between neck circumference and the risk of decreased estimated glomerular filtration rate in the general population of China: a cross-sectional study. Biomed Res Int. 2020;2020:3496328-11. https://doi.org/10.1155/2020/3496328.

31. Yan $P, X u$ Y, Miao $Y$, Bai $X$, Wu Y, Tang Q, et al. Association of remnant cholesterol with chronic kidney disease in middle-aged and elderly Chinese: a population-based study. Acta Diabetol. 2021. https://doi.org/10.1007/ s00592-021-01765-z Epub ahead of print. PMID: 34181081.

32. Virani SS, Smith SC Jr, Stone NJ, Grundy SM. Secondary prevention for atherosclerotic cardiovascular disease: comparing recent US and European guidelines on dyslipidemia. Circulation. 2020;141(14):1121-3. https://doi. org/10.1161/CIRCULATIONAHA.119.044282.

33. Ito S, Nagasawa T, Abe M, Mori T. Strain vessel hypothesis: a viewpoint for linkage of albuminuria and cerebro-cardiovascular risk. Hypertens Res. 2009; 32(2):115-21. https://doi.org/10.1038/hr.2008.27.

34. Huang MJ, Wei RB, Zhao J, Su TY, Li QP, Yang X, et al. Albuminuria and endothelial dysfunction in patients with non-diabetic chronic kidney disease. Med Sci Monit. 2017;23:4447-53. https://doi.org/10.12659/MSM.903 660.

35. Shankar A, Klein R, Klein BE. The association among smoking, heavy drinking, and chronic kidney disease. Am J Epidemiol. 2006;164(3):263-71. https://doi.org/10.1093/aje/kwj173.

36. Yokoyama H, Araki S, Haneda M, Matsushima M, Kawai K, Hirao K, et al. Chronic kidney disease categories and renal-cardiovascular outcomes in type 2 diabetes without prevalent cardiovascular disease: a prospective cohort study (JDDM25). Diabetologia. 2012;55(7):1911-8. https://doi.org/10.1 007/s00125-012-2536-y.

\section{Publisher's Note}

Springer Nature remains neutral with regard to jurisdictional claims in published maps and institutional affiliations. 